\title{
EVALUATION OF OUTCOMES OF ACUTE HAEMATOGENOUS OSTEOMYELITIS IN CHILDREN FOLLOWING SURGICAL DECOMPRESSION
}

\author{
Lamichhane A P*, Sharma C M*, K.C. G*, Shah L L*
}

\section{ABSTRACT}

Outcomes of the surgical decompression in 21 patients of less than 15 years of age with acute haematogenous osteomyelitis attending Tribhuvan University Teaching Hospital during the period Oct. 1998 to Sept. 2000 were evaluated prospectively. Average age of the patients was 9.8 years. (range 3-14 years.) Thirteen Patients were male and 8 were female. Majority $(71 \%)$ of the cases had involvement of the bones around the knee joint. Pus culture was positive in $(\mathbf{7 1 . 4 3 \%})$ of the cases. Among 21 cases who underwent surgical decompression, three patients were in inflammatory stage. Staphylococcus aureus was the causative organism in all culture positive cases and was sensitive to cephalexin in $86 \%$ and Cloxacillin in $100 \%$ of the cases. $X$ ray changes of chronic osteomyelitis developed in the $2^{\text {nd }}$ week in most cases $(70 \%)$. Overall rate of conversion to chronic osteomyelitis was $\mathbf{4 7 . 6 2 \%}$. Chronic osteomyelitis developed more in patients aged $>10$ years of age $(\mathrm{P}=\mathbf{0 . 0 0 1 5})$. Also chronic osteomyelitis developed more in patients who underwent surgical decompression after 10 days $(\mathrm{P}=\mathbf{0 . 0 6 3 4})$. The study concluded that early surgical decompression prevents chronicity and prognosis is better in young children.

Key Words: Acute haematogenous osteomyelitis, Children, Chronic osteomyelitis, Surgical decompression.

\section{INTRODUCTION}

Acute haematogenous osteomyelitis, infection of bone by pyogenic organism is almost invariably a disease of children. Due to various factors patients with acute haematogenous osteomyelitis present late to the hospital leading to delayed treatment and increased complication rate . Even with advent of antibiotics and development of sophisticated treatment modalities, it remains one of the most challenging problems for the orthopedic surgeons. ${ }^{1}$ The availability of an assortment of antibiotics has not lessened the need for critical evaluation of present concept of the treatment of osteomyelitis. Infact it actually has prompted a close analysis of the optimal treatment programme. The major controversy regarding the management of osteomyelitis centers on indications and advantages of surgical versus nonsurgical intervention in the acute stage. In the very early stages however, when recognition is difficult and there are no roentgenographically detectable bony changes, the role of surgical treatment is controversial. ${ }^{2}$

Many would avoid surgical treatment in the acute stages, claiming that it offers no added advantages over antibiotic therapy ${ }^{3}$ but others think that decompression is an essential factor in the early stages of the disease. ${ }^{4,5,6}$ Following surgical decompression some cases resolve without any complications and some are complicated with chronic osteomyelitis and other complications.

* Tribhuvan University Teaching Hospital, Maharajgunj, Kathmandu, Nepal.

Address for correspondence : Dr. Arjun P. Lamichhane

Dept. of Orthopaedics

Tribhuvan University Teaching Hospital, Maharajgunj, Kathmandu, Nepal.

Email: lamichhanearjun@hotmail.com 
So this study primarily focuses on analysis of outcome of acute haematogenous osteomyelitis in terms of chronic osteomyelitis, rate of conversion and factors influencing its conversion to chronic osteomyelitis following surgical intervention.

\section{MATERIALS AND METHODS}

This prospective study was conducted in the department of Orthopedic and trauma surgery, Tribhuvan University Teaching Hospital, Institute of Medicine, Maharajgunj, Kathmandu from October 1998 to September 2000.

The study consisted of 21 consecutive and eligible patients with acute haematogenous osteomyelitis who underwent surgical decompression.

\section{Inclusion criteria}

All Patients aged less than 15 years of age with acute haematogenous osteomyelitis (AHOM) without bones changes. Acute haematogenous osteomyelitis patients involving limb bones only.

\section{Exclusion criteria}

Any Patients of 15 years or older age.

Patients with acute exacerbation of chronic osteomyelitis (COM) or AHOM with chromic osteomyelitis changes.

Any exogenous osteomyelitis.

Clinical diagnosis of AHOM was made on the basis of history, physical examination and laboratory Parameters.

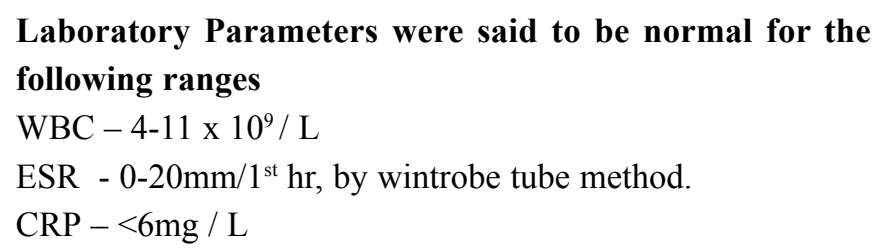

\section{Indications for Operation}

(1) Patients presenting within 72 hours of illness but not responding with appropriate intravenous antibiotic
(Cefazoline $100 \mathrm{mg} / \mathrm{kg} /$ day) for 48 hours or (2) Patients presenting after 72 hours of onset of illness.

Surgical decompression was done with standard technique. Pus was sent for culture and sensitivity.

Post-operative antibiotic was given intravenous for one week then oral cephalexin $50 \mathrm{mg} / \mathrm{kg} /$ day or according to culture and sensitivity for 6 weeks.

Patients were discharged on the $10^{\text {th }}$ day after removal of stitches.

Routine posterior slab was applied for lower limb bone and weight bearing was allowed as tolerated.

Follow-up was done on $2^{\text {nd }}$ week, $6^{\text {th }}$ week, $12^{\text {th }}$ week $\& 24^{\text {th }}$ week to see the outcome as COM.

Weight bearing was advised according to the extent of involvement by chronic osteomyelitis.

During each visit - clinical examination, Laboratory \& X-ray examinations were done to see disease activity \& COM changes.

\section{Statistical Analysis}

Results were analyzed by Fischer's exact Probability test using epi Info version 6 soft ware programme. We tried to correlate different variables taking confidence level at $95 \%$.

\section{OBSERVATION AND RESULTS}

There were 21 Patients of AHOM who needed surgical decompression with 13 males and 8 females.

- COM developed in the Patients of $>10$ years age (42.86\%) with significant $\mathrm{P}^{\prime}$ value $(\mathrm{P}=0.0075)$

- Conversion rate of AHOM to COM was 47.62\% (10 of 21 cases).

- In seventy-one percent of the cases in this series bones around the knee joint were involved.

- History of trauma Preceeding the illness was found in $33 \%$ (7) cases ,among them 3 developed COM.

Table I : Age and Development of COM 